\title{
Challenges In Telemedicine Implementation For Pregnancy Care During The Covid-19 Pandemic
}

\author{
Yuliana $^{1^{*}}$ \\ ${ }^{1}$ Fakultas Kedokteran, Universitas Udayana \\ *Email : lee.yuliana@ gmail.com
}

\begin{abstract}
Background: COVID-19 pandemic condition made the consultation shifted from face to face consultation to telemedicine for mild, stable, and follow up cases. In stabile condition of pregnant patients, telemedicine implementation will be beneficial to reduce the COVID-19 transmission risk. The problems are not all patients can implement telemedicine and the reimbursement procedure was hard. The aim of this paper is to describe about challenges in telemedicine implementation for pregnancy care during the COVID-19 pandemic. Methods: this paper is a literature review, Literature was identified from the archives of PubMed, Scopus, and Elsevier. All the literature was published in 2020. The inclusion criteria were the literature should be peer-reviewed and related to the topic of the paper. Keywords were challenges, COVID-19, opportunities, pregnancy, and telemedicine. Results: from 18 articles in this literature review, it was found that challenges in telemedicine are reimbursement, infrastructure, confidentiality, and unfamiliarity for the pregnant patients. Conclusion: Telemedicine can be used for pregnant patient control consultation during the stable condition to minimize the risk of COVID-19 transmission. The implication of this paper is to give insight that telemedicine can be done for stable pregnancy condition to reduce the risk of COVID-19 transmission during the pandemic. The benefits of reducing COVID-19 risk transmission for baby and mother outweigh the challenges and problems such as reimbursement, confidentiality, and technical issues.
\end{abstract}

Keywords: challenges, covid-19, opportunities, pregnancy, telemedicine

\section{INTRODUCTION}

The World Health Organization announced the coronavirus disease 2019 (COVID-19) as a pandemic on 11 March, 2020. The coronavirus disease spreads rapidly from Wuhan, China to the world. The management strategies were early identification, diagnosis, isolation, treatment, monitoring, and follow up with the patients. Health service is shifted partially into telemedicine (telehealth) to reduce transmission risk (Ohannessian et al., 2020).

Telemedicine refers to do healthcare services without direct meeting using Information and Communications Technology (ICT) for the treatment of patients. By telemedicine technology, medical information and services can be delivered to the patients without meeting directly (Lin et al., 2020). This technology can minimize exposure to possible COVID-19 cases (Smith et al., 2020; Vidal-Alaball et al., 2020). Telemedicine has two-way and live communication. It can be done from a distant site. It involves audio and video. High-resolution video and stable internet connection make telemedicine smooth (Barney et al., 2020). Telemedicine can be used to review patient medication histories and anamnesis (Bokolo, 2020). Telemedicine may be done without physical examination, but it will not replace inperson medical visits (Barney et al., 2020).

Low cost, safety, fast care, convenience, and easy to access are the positive sides of telemedicine. It is suitable for mild cases. When the distance is an essential factor, telemedicine is appropriate. In the COVID-19 pandemic, 
doctor and patient should maintain social and physical distancing, therefore, telemedicine served the best option for a medical communication tool. Health information exchange for diagnosis, treatment, follow-up, and prevention can be conducted by telemedicine. Follow-up is important for continuity of care (VidalAlaball et al., 2020). Telemedicine can make human resources usage more efficient. Personal protective equipment usage will be less if telemedicine is implemented. Telemedicine can be used at sources of health promotion for patients (Chersich et al., 2020).

Telemedicine types are online consultations (telephone or video conference), telemonitoring, sensors (to avoid possible dangerous location), and chatbots. Patients can report symptoms. Doctors can triage patients before they come to the emergency room. Chatbots are useful for recommendations and answering the usual questions (VidalAlaball et al., 2020). Telemedicine can be delivered by audio, video, or even textbased. However, video is better because of the ability to investigate very simple physical examinations such as in small cases of dermatology disorder. Low video quality makes physical examination difficult. The timing of telemedicine can be real-time or asynchronous. Nevertheless, real-time transmission telemedicine is preferred. Direct and realtime interaction is more efficient and time-saving to reach prompt diagnosis and treatment. The user of telemedicine are patient, health worker, or caregiver of the patient. About fifty percent of hospitals in the United States used telemedicine in the radiology department. Diagnosis can be done remotely through cloud-based imaging systems (Loeb et al., 2020).
Based on the research, telemedicine can be done at multiple locations. Telemedicine can reduce time in obtaining a diagnosis and initiating treatment. By home telemedicine, doctors and patients can reduce travel time and intrahospital infection (Vidal-Alaball et al., 2020). Telemedicine can reduce clinicians' burnout while dealing with patients face to face (Hare et al., 2020). Telemedicine saves medical protective equipment such as gloves and antiseptics (Vidal-Alaball et al., 2020). By telemedicine, the patients can avoid the inconvenience of traveling, seeking parking at the clinics and hospitals. Patients don't need to have a leave due to medical consultation (Contreras et al., 2020).

Pregnant patients can have a telemedicine consultation, especially those who are in stabile condition. They might minimize their post-partum hospital stays through remote monitoring from home (Mann et al., 2020). Patients in the isolation room can communicate with their doctors through telemedicine. Virtual telecommunication is important to establish a connection between patient and doctor whilst maintaining health precaution for COVID-19. This practice is essential in flattening the COVID-19 pandemic curve (Lin et al., 2020). The aim of this literature review is to describe about challenges in telemedicine implementation for pregnancy care during the COVID-19 pandemic.

\section{METHODS}

This paper is a literature review. Literature was identified from the archives from the data base of PubMed, Scopus, and Elsevier. All the literature was published in 2020. Keywords were challenges, COVID-19, opportunities, 
pregnancy, and telemedicine. There were 25 journals at the beginning. After using the inclusion criteria, there were 18 journals left. The inclusion criteria were the literature must be peer-reviewed and related to the topic of the paper. The exclusion criteria were view point journals. I analysed the data by categorizing the journals based on the topics, type of paper, and its relevancy.

\section{RESULTS}

There were 18 articles found in this literature review. It was found that challenges in telemedicine are reimbursement, infrastructure, confidentiality, and unfamiliarity for the pregnant patients. The suitable cases for telemedicine consultation are mild and stable condition such as follow up cases.

\section{DISCUSSION}

From 18 articles of the result, it was found that most countries have less regulation to integrate telemedicine services. France actively used telemedicine, while Italy excluded telemedicine within the National Health Service (Ohannessian et al., 2020). Trump had allowed Medicare-enrolled Americans to use telemedicine without extra cost (Vidal-Alaball et al., 2020). Telemedicine utilization was increased obviously after the pandemic (Contreras et al., 2020).

The Centers for Medicare and Medicaid service, also insurance companies have deleted restrictions in using telemedicine. This action will save health system resources (Khairat et al., 2020). There are 1135 waivers as temporary regulation for telemedicine during the COVID-19 pandemic. The waiver implemented the same reimbursement between telemedicine and regular visit. Regulation regarding outside state boundaries when implementing telemedicine is also waived by some states. Telemedicine is an important tool in board conferences among specialists all over the world (Contreras et al., 2020).

The government also funded for telemedicine by increasing broadband data capacity and software. A sum of $\$ 200$ million was given for low-income Americans. This money can increase internet speed connectivity and telemedicine hardware as needed. Recommended platforms for telemedicine during the COVID-19 pandemic are Cisco Webex Meetings/Webex Teams, and Google G Suite Hangout Meet. Meanwhile, Facebook Live, TikTok, Twitch, and other similar public-facing videos are strongly prohibited. In a period of 24 days, telemedicine visits increased dramatically from less than 100 to more than 2200 per day. A telephone call and video-based telemedicine can be used. However, a telephone call gives less information than a video call. The reimbursement billing level is also relatively lower than video consultation. Therefore, both doctors and patients are better to familiarize themselves with video consultation to have comprehensive consultation (Contreras et al., 2020).

Every country has a different strategy in implementing telemedicine. Australia has implemented telemedicine. Singapore made a tracing system to report the location of quarantine. The data were linked with the serological test result to show the transmission chain. The Seoul National University Hospital used telemedicine in the COVID-19 pandemic. In Spain, phone calls are used to monitor patients' symptoms. Patients can access their sick leave letter through electronic 
medical record systems (Vidal-Alaball et al., 2020).

Telemedicine

consultation included the real-time interaction between patient and doctor. Anamnesis, monitoring, and triage can be done through telemedicine. Technological resources and developments, credentialing, the high-speed internet connection, and high tech smartphones make video teleconsultations run smoothly from a patient's home (Loeb et al., 2020; Ohannessian et al., 2020).

A public academic hospital in Paris had encouraged outpatient teleconsultation since March 13, 2020. Funding, definition, clinical guidelines, standardized auto questionnaire, and remote patient-monitoring algorithms are needed as soon as possible. Data sharing systems integrated with epidemiological surveillance are needed to evaluate and research update conditions. While waiting for the integrating process, adoption of existing regulatory changes can be implemented (Ohannessian et al., 2020).

Mann et al. did a study from March 2nd until April 14th, 2020 in NYU Langone Health at New York City in the United States. They found that telemedicine visits had a $135 \%$ increase in urgent care and a $4345 \%$ increase in nonurgent care. Among those data, 56.2\% in urgent care and $17.6 \%$ in non-urgent care were related to COVID-19. American Telemedicine Association stated that telemedicine is useful for mild cases (Mann et al., 2020).

Telemedicine can minimize COVID-19 risk transmission and manage thousands of patients in a short time. This technology seems comfortable for patients and doctors. Pregnant patients might minimize their post-partum hospital stays through remote monitoring from home (Mann et al., 2020). Patients in the isolation room can communicate with their doctors through telemedicine. Virtual telecommunication is important to establish a connection between patient and doctor whilst maintaining health precaution for COVID-19. This practice is essential in flattening the COVID-19 pandemic curve (Lin et al., 2020).

Improving telemedicine technology is a good practice to deal with acute, chronic, and preventive care in the COVID-19 pandemic era and post COVID-19 pandemic. Lin et al (2020) showed that telemedicine reduced the time exposure of patients with COVID-19 risk of infection without reducing patient satisfaction. They did a retrospective study of 198 patients in National Taiwan University Hospital from the beginning of March to the end of April 2020. The patients were divided into a telemedicine group and a direct meeting consultation group. Five-point Likert scale questionnaires were used to assess patients' satisfaction. Length of evaluation time, stress perception, and privacy were evaluated. The telemedicine group revealed a shorter evaluation period time compared to the conventional group (4.7 minutes vs 8.9 minutes, respectively). This result had some confounding factors such as patient characteristics and laboratory examination. Using telemedicine in triage possible COVID-19 cases could minimize the workload of health care workers in dealing with suspected COVID-19 patients. The health workers could more concentrate on treating critical patients. However, this result might vary between countries, other cultural societies, and different age groups. Telemedicine must be used only 
for stable and non-critical suspected COVID-19 patients. Identity, general appearance, and respiratory status can be reviewed through video call consultation. The physician can send laboratory data and images with good quality to the patient simultaneously (Lin et al., 2020).

Patients don't need to drive long distances. They are free from searching parking lots in the middle of busy hospital parking. Money to buy gasoline can be saved to buy internet data. Instead of wasting time waiting for doctors' queues, the patients can browse useful health information on the internet (Szperka et al., 2020).

\section{Challenges In Implementing Telemedicine During The COVID-19 Pandemic}

Starting telemedicine is difficult when the integration system of data is not connected. However, integrating an effective telemedicine system cannot be settled and arranged in one day. It needs time. This poor interconnection data integration is usually accompanied by a limited reimbursement system. Unfamiliarity with telemedicine technologies is the most common challenge in telemedicine applications. Doctors should update their skills in diagnosis and counseling by using telemedicine. Meanwhile, patients must be trained on how to update their biometric data such as temperature, blood pressure, and glucose level (Mann et al., 2020).

Reimbursement and funding can arouse serious matter for telemedicine implementation. The Australian government funding for Medicare telehealth was distributed based on geographical location and health service type. The priority is for videoconference medical consultation by the patients in the village. On the other hand, metropolitan people cannot have the same funding privilege. The most common risk for COVID-19 transmission is in the areas with greater population density, i.e. city location. Priority funding for elderly people with a respiratory illness will help a lot in the COVID-19 pandemic. The federal government in the United States of America gave funding for patients in the metropolitan area to access telemedicine. International foundations such as the World Health Organization advocated the use of telemedicine on the website (Smith et al., 2020).

Confidentiality, quality of care, patient acceptance, and connectivity are the crucial problems in using telemedicine. Privacy can be managed by using the Zoom chat feature. Families at home won't hear the conversation when the earphone is used. Closed questions with yes or no answers are the best solutions for confidentiality. The sensitive examination must be well handled. Photo upload can be used instead of showing the sensitive part in online telemedicine (Barney et al., 2020). The password should be made unique for each consultation so only the doctor and the patient know the password code. This will minimize the unsecured connection (Smith et al., 2020).

Relaxation regulation restriction will help physicians to take care of their patients from home. It is time-saving for physicians. Australia implemented a similar regulation. A comprehensive system strategy is needed to perform telemedicine smoothly. This involves operational telemedicine networks, technology infrastructure, telemedicine 
policy, and flexible regulation (Smith et al., 2020).

Clinical-decision making will be limited without a proper physical examination and/or laboratory examination. This can be managed by a nursing visit for laboratory examination procedures. Clinical scoring is useful to sharpen the possible diagnosis. Some questionnaires are better to send before starting telemedicine. This can make consultation more effective using telemedicine. A nurse can help patients to measure vital signs, visual acuity, and weight. Vaccination and injection are procedures that can be done at home by a nursing visit. Contraceptive consultation (pills) can be made through telemedicine. However, other reproductive cases (sexually transmitted diseases, pelvic inflammatory diseases, or HIV tests, intrauterine devices, injection, implant insertion) require physical examination (Barney et al., 2020).

From the patients' point of view, there are some barriers to use telemedicine. Patients should measure their weight, blood pressure, temperature, respiratory rate, and pulse. Procedures such as vaccination, phlebotomy, and laboratory examination can't be done with telemedicine only (Barney et al., 2020). Limited internet connectivity can make telemedicine difficult (Chersich et al., 2020).

Privacy problems arise when telemedicine is implemented using free apps such as WhatsApp, Skype, Zoom, FaceTime (Ohannessian et al., 2020). To integrate the patient data with the public health authority need time and funding. While the process takes time, it's recommended to apply the available platforms. The practical recommendation has to be prepared. The development of software, hardware clinical validation, and certification must be performed for telemedicine. We must not delay the telemedicine implementation to flatten the COVID-19 pandemic transmission curve (Portnoy et al., 2020).

There are administrative issues to be addressed during telemedicine. Doctors must have a license based on the patient's location at the time of telemedicine done. Insurance coverage is not always available for telemedicine. Confidentiality can't be guaranteed when using free platforms such as Zoom, WhatsApp, etc. Vaccination, laboratory test, medicine prescription, or physical examination cannot be fulfilled through telemedicine (Wright \& Caudill, 2020).

The health laws for telemedicine are different in every state. Qualified doctors are allowed to practice in any state according to federal waiver restraints. State laws prohibit doctors to do new patients consultations and prescriptions accordingly. If telemedicine is not applicable, then a telephone call can be done. A telephone call is reimbursed in less money than video consultation (Szperka et al., 2020).

Telemedicine is an effective tool to help the patient during the COVID-19 pandemic. Remotely lived patients can easily have medical consultation by telemedicine. However, when emergency case arises, patients need to seek nearest hospital as soon as possible (Szperka et al., 2020).

\section{Procedure In Performing Telemedicine}

The doctor should remind the patients about the possibility of cybersecurity risk. The doctor must dress professionally. Eye contact should be 
maintained during telemedicine. Lighting and sound have to be clear to prevent misunderstanding. Doctors have to speak slowly and briefly. Every patient's question must be addressed appropriately.

The doctor should give the patient some checklist preparations before telemedicine begins. The doctors must introduce his/her name. The patient's data must be verified before the telemedicine session is begun (Contreras et al., 2020). Checklist preparation includes biometric measurement, the procedure to share photos, how to use a camera and microphone properly during a consultation (Bokolo, 2020).

The whole telemedicine procedure needs the collaboration of all clinic staff members (Barney et al., 2020). Mode of telemedicine (by phone, email, or video), length of consultation, the reason for a doctor consultation, medical record, and doctor recommendation must be documented. How many minutes the conversation take place have to be written (Contreras et al., 2020).

Telemedicine can be used to triage possible COVID-19 cases. Anamnesis about exposure risk and observational assessment can be done. Temperature, respiratory rate, and breath quality can be assessed. The presence of a cough with oropharyngeal assessment can be performed (Portnoy et al., 2020). However, complete and comprehensive physical examination cannot be done through telemedicine. Emergency cases, patients in critical condition, and difficult cases need to have in-person visits. Auscultation, percussion, and palpation are difficult to perform virtually (Smith et al., 2020).

\section{Practical Implication For Pregnant Patient In Implementing Telemedicine Consultation}

Current practice for prenatal visits are every 4 weeks until 28 weeks of gestational age. After that, it is recommeneded every 2 weeks until 36 weeks of gestational age. Finally, prenatal visit must be done once weekly until delivery. However, during the COVID-19 pandemic, there should be combination from virtual visit (telemedicine) and in person visit. In person visit is useful for physical and ultrasound examination (Fryer et al., 2020).

Suitable cases for the telemedicine consultation in pregnant patients are the stabile condition and the low risk pregnancy. Recommended cases for telemedicine are missed period, confirmation of pregnancy, ante natal care without any complication or comorbid diseases of the mother, urinary tract infection, and vaginal itching/irritation. However, some emergency and high risk cases have to do face to face consultation. Those cases are uncontrolled diabetes and hypertension in pregnancy, any signs and symptoms of bleeding, pain, less movement, miscarriage, watery discharge per vaginum, antepartum hemorrhage, pre-eclampsia, vaginal bleeding, suspected torsion, suspected ectopic pregnancy, acute pelvic inflammatory disease, and preterm labour (Bindra, 2020).

Pregnant patient must be taught to observe and count the daily movement of her baby. Leaking of watery discharge per vaginal is an emergency sign that need to be evaluated in the hospital. While during the COVID-19 pandemic, the mother has to do hand washing and social distancing often. Non urgent consultation can be 
done based on individual case (Bindra, 2020). Telemedicine will help pregnant women to monitor their baby condition without compromising on the perinatal outcome (Sharmila et al., 2020).

The simple physical examination such as measuring body weight and blood pressure can be done by the pregnant patient at home. Fundal height is measured by caretaker after seeing demonstration video from the doctore. Home monitoring for blood glucose can be done also by the controlled diabetic pregnant patient. Anemia can be seen through pallor of conjunctiva by video call. Patient can be taught how to prepare for lactation after delivery. If there is any case of emergency, patient must contact the doctor or go to the emergency department as soon as possible (Sharmila et al., 2020).

Postpartum consultation can be performed for breastfeeding problems, contraceptive needs, and follow up the wound after caesarean delivery. Postpartum visit must be done at within three to six weeks after delivery (Sharmila et al., 2020). Performing postpartum consultation by telemedicine is useful to reduce to risk of COVID-19 transmission and postpartum complication (Reynolds, 2020).

Postpartum mother can suffer from mental problems such as depression and psychosis. Cognitive behavioral therapy for those disorders can be delivered through telemedicine. However, when the patients had worsening symptoms with life threatening signs (such as suicidal thought), they must directly come to the hospital (Sharmila et al.,2020).

Newborn baby care can be assessed through telemedicine. Any data of adequate feeding, urine output, crying, and the activity of the baby can be obtained by the mother at home. The data are reported to the doctor by telemedicine. This practice will reduce the risk of COVID-19 transmission for the babies (Sharmila et al., 2020).

\section{CONCLUSION AND SUGGESTIONS}

Telemedicine is very useful to minimize COVID-19 spread during a consultation for patients and doctors. Phone and videoconferencing can be chosen for mild and stable cases. Medical supplies will be saved when using telemedicine. Triage can be done by telemedicine so the emergency department will not be overwhelmed by the patient's queue. Confidentiality remains a crucial thing to be addressed.

Patients must be recommended to perform telemedicine in a private place to assure confidentiality. Suitable pregnancy cases for telemedicine consultation are the low risk pregnancy without any complication and comorbid previously. However, the emergency signs are any pain, bleeding, decreasing of baby movement, or any discharge per vaginam. Postpartum follow up and postpartum complication can be assessed through telemedicine. Adaptation and flexibility to change the conventional consultation to telemedicine based on the case urgency and suitability is a must during the COVID-19 pandemic. Telemedicine will help pregnant women to monitor their baby condition without compromising on the perinatal outcome.

\section{REFERENCES}

Barney, A., Buckelew, S., Mesheriakova, V., \& Raymond-flesch, M. (2020). The COVID-19 Pandemic and Rapid Implementation ofAdolescent 
and Young Adult Telemedicine: Challenges and Opportunities for Innovation. 1-8.

Bindra, V. (2020). Telemedicine for Women's Health During COVID 19 Pandemic in India: A Short Commentary and Important Practice Points for Obstetricians and Gynaecologists. The Journal of Obstetrics and Gynecology of India, 70(4), 279-282. https://doi.org/10.1007/s13224-02001346-0

Bokolo Anthony Jnr. (2020). Use of Telemedicine and Virtual Care for Remote Treatment in Response to COVID-19 Pandemic. Journal of Medical Systems, 44(7). https://doi.org/10.1007/s10916-02001596-5

Chersich, M. F., Gray, G., Fairlie, L., Eichbaum, Q., Mayhew, S., Allwood, B., English, R., Scorgie, F., Luchters, S., Simpson, G., Haghighi, M. M., Pham, M. D., \& Rees, H. (2020). COVID-19 in Africa: care and protection for frontline healthcare workers. Globalization and Health, 16(1), 46. https://doi.org/10.1186/s12992-02000574-3

Contreras, C. M., Metzger, G. A., Beane, J. D., Dedhia, P. H., Ejaz, A., \& Pawlik, T. M. (2020). Telemedicine: Patient-Provider Clinical Engagement During the COVID-19 Pandemic and Beyond. Journal of Gastrointestinal Surgery, 24(7). https://doi.org/10.1007/s11605-02004623-5
Fryer, K., Delgado, A., Foti, T., Reid, C. N.,\&Marshall,J.(2020).Implementati on of Obstetric Telehealth During COVID - 19 and Beyond. Maternal and Child Health Journal,17.https://doi.org/10.1007/s10995020-02967-7

Khairat, S., Meng, C., Xu, Y., Edson, B., \& Gianforcaro, R. (2020). Interpreting COVID-19 and Virtual Care Trends: Cohort Study. JMIR Public Health and Surveillance, 6(2), e18811.https://doi.org/10.2196/1881 1

Lin, C. H., Tseng, W. P., Wu, J. L., Tay, J., Cheng, M. T., Ong, H. N., ... Chen, S. C. (2020). A Double Triage and Telemedicine Protocol to Optimize Infection Control in an Emergency Department in Taiwan During the COVID-19 Pandemic: Retrospective Feasibility Study. Journal of Medical Internet Research, 22(6), e20586. https://doi.org/10.2196/20586

Loeb, A. E., Rao, S. S., Ficke, J. R., Morris, C. D., Riley, L. H., \& Levin, A. S. (2020). Departmental Experience and Lessons Learned With Accelerated Introduction of Telemedicine During the COVID-19 Crisis. The Journal of the American Academy of Orthopaedic Surgeons, 28(11), e469-e476. https://doi.org/10.5435/JAAOS-D20-00380

Mann, D. M., Chen, J., Chunara, R., Testa, P. A., \& Nov, O. (2020). COVID-19 transforms health care 
through telemedicine: evidence from the field. Journal of the American Medical Informatics Association: JAMIA.https://doi.org/10.1093/jamia locaa072

Ohannessian, R., Duong, T. A., \& Odone, A. (2020). Global Telemedicine Implementation and Integration Within Health Systems to Fight the COVID-19 Pandemic: A Call to Action. JMIR Public Health and Surveillance, 6(2), e18810. https://doi.org/10.2196/18810

Portnoy J., Waller M, Elliot, T. (2020). Telemedicine in the Era of COVID19.J Allergy Clin Immunol Pract., 8(5):148991.https://doi.org/10.1016/ j.jaip.2020.03.008. (2020).

Reynolds, R. M. (2020). Telehealth in pregnancy. THE LANCET Diabetes \& Endocrinology, 8(6), 459-461. https://doi.org/10.1016/S22138587( 20)30158-3

Sharmila, V., Babu, T. A., \& Balakrishnan, P. (2020). Optimizing telemedicine for providing obstetric and reproductive health care services during COVID-19 pandemic.7(8),

32783283.http://dx.doi.org/10.1820

3/2394-6040.ijcmph20203414

Smith, A. C., Thomas, E., Snoswell, C. L., Haydon, H., Mehrotra, A., Clemensen, J., \& Caffery, L. J. (2020). Telehealth for global emergencies: Implications for coronavirus disease 2019 (COVID19). Journal of Telemedicine and Telecare,26(5),309313.https://doi.or $\mathrm{g} / 10.1177 / 1357633 \mathrm{X} 20916567$

Szperka, C. L., Ailani, J., Barmherzig, R., Klein, B. C., Minen, M. T., Halker Singh, R. B., \& Shapiro, R. E. (2020). Migraine Care in the Era of COVID-19: Clinical Pearls and Plea to Insurers. Headache, 60(5), 833842.https://doi.org/10.1111/head.13 810

Vidal-Alaball, J., Acosta-Roja, R., Pastor Hernández, N., Sanchez Luque, U., Morrison, D., Narejos Pérez, S., ... Salvador Vèrges, A. (2020). Telemedicine in the face of the COVID-19 pandemic. Atencion Primaria, 52(6), 418-422. https://doi.org/10.1016/j.aprim.2020 .04 .003

Wright, J. H., \& Caudill, R. (2020). Remote treatment delivery in response to the COVID-19 Pandemic. Psychotherapy and Psychosomatics, 89(3), 130-132. https://doi.org/10.1159/000507376 